\title{
Low-redshift quasar absorption-line systems: inside and around galaxies
}

\author{
Weipeng Lin ${ }^{1}$, G. Börner ${ }^{2}$, and H. J. Mo ${ }^{3}$ \\ ${ }^{1}$ The Partner Group of MPA, Shanghai Astronomical Observatory, Nandan Road 80, \\ Shanghai 200030, China, email: linwp@shao.ac.cn \\ ${ }^{2}$ Max-Planck Institute for Astrophysics, Karl Schwarzschild Str. 1, Postfach 1317, \\ 85741 Garching, Germany, email: grb@mpa-garching.mpg.de \\ ${ }^{3}$ Department of Astronomy, University of Massachusetts, Amherst MA 01003-9305, USA \\ email: hjmo@vela.astro.umass.edu
}

\begin{abstract}
We present semi-analytic models and Monte-Carlo simulations of QSO Ly $\alpha$ absorption line systems which originate in gaseous galactic halos, galaxy disks, and satellite halos around central galaxies. The relation between the properties of predicted absorption lines and those of galaxies has been investigated. Using a model with galactic halos, satellites, and HI disks of spirals, $60 \%$ of the observed strong Ly $\alpha$ lines and all of the Lyman-limit systems can be predicted. The models predict $W_{r} \propto \rho^{-\alpha} L_{B}^{\beta}(1+z)^{-\gamma}$ with $\alpha \sim 0.5, \beta \sim 0.15, \gamma \sim 0.5$. For strong Ly $\alpha$ lines the average covering factor within $250 h^{-1} \mathrm{kpc}$ is $\sim 0.36$, with an average absorption radius of $\sim 150 \mathrm{~h}^{-1} \mathrm{kpc}$ for a galaxy halo, in good agreement with observations. Using mock imaging and spectroscopic surveys, selection effects are found to be able to strengthen the anti-correlation between line width and projected distance. The models were also applied to quasar metal absorption lines and more realistic absorption spectra with noise were simulated.
\end{abstract}

\section{Introduction}

Why study the possible relation between quasar absorption line systems and luminous galaxies? From a theoretical point of view, there are two main reasons. Firstly galactic dark matter halos contain lots of multiphase gas, some of which can only be probed through quasar absorption lines. Secondly the gas processes inside galactic halos are very important for galaxy formation, and therefore investigation of absorption lines can help to find crucial clues to and set constraints on galaxy formation theories. However, the origin and nature of low redshift quasar absorption lines are still a matter of debate, even though it is of no doubt that some absorption line systems are related to luminous galaxies. Several questions remain. For example, do quasar absorption line systems arise from extended gaseous galactic halos or from the diffuse intergalactic medium? What is the nature of the absorber-galaxy connection? Which parts of the galaxies (or halos) can give rise to absorption line systems? Are there any effects of environment (e.g. galaxy groups, clusters, or voids) on the quasar absorption lines? Tremendous efforts have been made to identify the galaxies giving rise to QSO absorption and to analyse their physical properties (see other papers in this conference). For instance, Lanzetta et al. (1995) and Chen et al. (1998) claimed that luminous galaxies can account for at least 50 percent (or even more) of the strong Ly $\alpha$ absorption lines $\left(W_{r} \geqslant 0.3 \AA\right)$ and a distinct anti-correlation of REW versus projected distance was established (see also Impey, Petry \& Flint 1999). Luminous galaxies are found to possess extended gaseous envelopes of $\approx 170 h^{-1} \mathrm{kpc}$ in radius. Tripp et al. (1998) cautioned that selection effects could artificially tighten the anti-correlation because galaxy surveys could be incomplete. At the same time, many 
theoretical efforts have been made to relate absorption systems to galaxies (see references in Lin, Börner \& Mo 2000, and other papers in this conference). This conference paper will mainly introduce previous work based on the papers by Lin, Börner \& Mo (2000) and Lin \& Zou (2001). In the models, galaxy disks, gaseous halos, and satellite halos can give rise to absorption lines. A $\Lambda$ CDM cosmogony (with $\Omega_{0}=0.3, \Omega_{\Lambda, 0}=0.7, h \equiv$ $\left.H_{0} / 100 \mathrm{~km} \mathrm{~s}^{-1} \mathrm{Mpc}^{-1}=0.7\right)$ was assumed.

\section{Key ingredients of the models and methods}

Monte-Carlo galaxy samples were generated from the luminosity function (LF) of field galaxies, where we use the AOTUFIB LF in Schechter form with $\phi^{*}=2.45 \times$ $10^{-2} h^{3} \mathrm{Mpc}^{-3}, M_{B *}=-19.30$, and $\alpha=1.16$ (Ellis et al. 1996). The cut-off at the faint-end was $0.01 L_{B *}$. These sample galaxies were placed within a cylindrical volume of radius $R_{\text {cy }}=400 h^{-1} \mathrm{kpc}$ in co-moving coordinates along the line-of-sight (LOS) toward a given QSO at redshift $z_{q}$. The number of galaxies at redshift $z$ within an interval $\Delta z$ is $\Delta N_{\mathrm{g}}=n_{c}(1+z)^{3} \pi R_{\mathrm{cy}}^{2} c(d t / d z) \Delta z\left(0<z<z_{\mathrm{q}}\right)$. The co-moving galaxy density $n_{c}$ is the integral of the LF, and the galaxy luminosities were generated in such a way that their distribution is consistent with the LF. A halo can be parameterised by its circular velocity $V_{\text {cir }}$, which is simply related to galaxy morphological type and luminosity. Empirical relations are known between luminosity and LOS velocity dispersion for E/S0 galaxies (Faber-Jackson Law, Faber \& Jackson 1976) and velocity spread for spirals (Tully-Fisher Relation, Tully \& Fisher 1977). The apparent magnitude of a galaxy was calculated by applying a k-correction and cosmological dimming.

Given $V_{\text {cir }}$, the gaseous galactic halo was modelled following Mo \& Miralda-Escudé (1996). It assumed that the gas in a halo has a two-phase structure (a hot and a cold phase) which in principle is described by the density profiles and the temperature profile. When the hot gas (assumed isothermal) starts to cool, it will form cold gas and sink to the galaxy centre, whose mass and distribution can be derived by using a cooling flow model. For simplicity, the cold gas was assumed to be in spherical clouds whose mass distribution is in the form of a log-normal with a mean mass of $5 \times 10^{5} M_{\odot}$. These clouds (with a temperature of $2 \times 10^{4} \mathrm{~K}$ ) were confined by pressure from the hot medium with the virial temperature. Therefore the cloud radius and the total hydrogen column density through the cloud centre can be calculated easily (see Lin, Börner \& Lin 2000 for more details). These clouds were assumed to be photo-ionised by a constant UV background, and in ionisation equilibrium. The fraction of hydrogen in the neutral state (HI atoms), is determined by the flux of the UV background ionisation field $J(\nu)$ (Miralda-Escudé \& Ostriker 1990; Madau 1992) and $n_{\mathrm{H}}$. The column density for neutral hydrogen $N_{\mathrm{HI}}$ can be derived using the code CLOUDY (Ferland 1996). We can also derive a column density for ion $\mathrm{X}$ (which can be $\mathrm{Mg}$ II, Fe II, Si II, etc.) from the ratio of $N_{X} / N_{\mathrm{HI}}$ as a function of ionisation parameter $U$. Then we obtain the column density $N_{X}$ of a cloud at a distance to the $\operatorname{LOS} l, N_{X}=N_{X}(0) \sqrt{1-l^{2} / R_{c}^{2}}$, where $N_{X}(0)$ is the column density through the cloud centre.

The results of N-body simulations provide us with a satellite population around a big galaxy-size halo (Klypin et al. 1999). The velocity dispersion of satellites is of the order of the circular velocity of the central halo. The spatial distribution of satellites around the central galaxy is assumed to follow an inverse square law. The column density of HI in these satellite halos can be derived following Mo \& Morris (1994).

We model the galaxy disk as a thin disk whose gas is ionised by the cosmic UV ionising field incident from the both sides. The HI in such a disk has a "sandwich structure", that is, in the central parts of disk, almost all the hydrogen atoms are in the HI phase, and 
there is a HII region above the HI layer. This HI layer extends out to some distance, where the HI column density then drops sharply and the HI layer disappears (namely "the sharp edge" of a HI disk). The disk HI column density has some dependence on the inclination and orientation angle of the LOS, which can be calculated in a straight-forward manner (see Lin \& Hong 2002 for details).

As we know the HI column density, again the column density for ion X can be calculated using CLOUDY. There might be one or more absorbing clouds in the LOS with different velocities with respect to the galaxy centre. Assuming a Voigt profile for each absorbing component, the optical depth of a single line is $\tau_{\nu} \approx 2.65 \times 10^{-2} f_{j k} N_{j} \phi\left(\nu ; \nu_{j, k}\right)$ for $h \nu_{j k} \ll k T$, where $f_{j k}$ is the oscillator strength of the line, $N_{j}$ is the column density, $\phi$ is the Voigt profile, and $j, k$ are the lower and upper energy level indices respectively. The rest frame equivalent width $(\mathrm{REW})$ is defined in frequency units is $W=\int\left(1-e^{-\tau_{\nu}}\right) d \nu$. In accordance with observational usage, $W$ is defined in wavelength units, so the value must be multiplied by $\lambda / \nu$. Integrating over the velocity structure due to the multi-absorbing clouds, the overall REW can be calculated.

\section{Strong Ly $\alpha$ lines $(\mathrm{REW} \geqslant 0.3 \AA ̊)$}

We performed Monte-Carlo simulations with 200 lines of sight, each of which covers a redshift span from 0 to 1 . Five sub-models (each of which has some free parameter space) were used to study the contributions of absorbing components for strong Ly $\alpha$ lines (see Lin et al. 2000). In summary, at $z<1$, galaxy disks are adequate to explain all DLAs (however, in fact not all DLA host galaxies are spirals), and cold clouds in gaseous galactic halos can explain about $20 \%$ strong Ly $\alpha$ lines. In addition gaseous satellite halos aroung big halos can explain up to $45 \%$ of strong Ly $\alpha$ lines. In total, a full model with all the three components can predict $d N / d z \sim 12$ and explain about $60 \%$ of strong Ly $\alpha$ lines (and also predict all Lyman limit systems, $d N / d z \sim 0.7$ ), and this fraction is $90 \%$ if the ionising field can be reduced by some factor (but predicts too many Lyman limit systems). For the "standard" model, the REW distribution has a peak near $0.3 \AA$ and the majority of REWs fall between 0.1 and $1.0 \AA$; about $70 \%$ of the projected distances of absorbers are between 20 and $200 h^{-1} \mathrm{kpc}$, only $10(20) \%$ of the absorbers have projected distances less than $20 h^{-1} \mathrm{kpc}$ (larger than $200 h^{-1} \mathrm{kpc}$ ); about $70 \%$ of the luminosities of the absorbing galaxies are between 0.1 and $1.0 L_{B *}$, which implies that absorbers are relatively luminous galaxies in the model.

We also investigated the relation between $\operatorname{REW}\left(W_{r}\right)$ and projected distance $(\rho)$, luminosity $\left(L_{B}\right)$, and redshift $(z)$, as well as the average covering factor and absorbing radius. Let $\alpha, \beta, \gamma$ be the coefficients of the relation between $\log W_{r}$ and $\log \rho, \log L_{B} / L_{B *}$, and $\log (1+z)$ respectively. The relation between $W_{r}$ and $\rho$ is $\alpha \sim 0.4-0.7$ for the full models, while for the relation between $W_{r}$ and $\rho$ together with $\log L_{B} / L_{B *}, \alpha \sim 0.5-0.8, \beta \sim 0.20$. In general, for the relation between REW and all the rest, $\alpha \sim 0.5-0.8, \beta \sim 0.20$, and $\gamma \sim 0.5-0.6$. From the analysis above, the average covering factor and the absorbing radius within $250 h^{-1} \mathrm{kpc}$ are estimated to be 0.36 and $150 h^{-1} \mathrm{kpc}$ respectively. The observational results are 0.3 and $174 h^{-1} \mathrm{kpc}$ respectively (Lanzetta et al. 1995; Chen et al. 1998). These results of our models are in good agreement with observations. In the models, spiral galaxies can give rise to only $35 \%$ of simulated strong Ly $\alpha$ lines while the rest arise from E/S0 galaxies. This fraction can be used to set constraints on models but it is not clear in observations to date.

Selection effects are very important in optical surveys of absorber-galaxy pairs. Under the limitation of telescope ability, in optical surveys of luminous absorbing galaxies one links an absorption line to the nearest luminous galaxy which has approximately the 

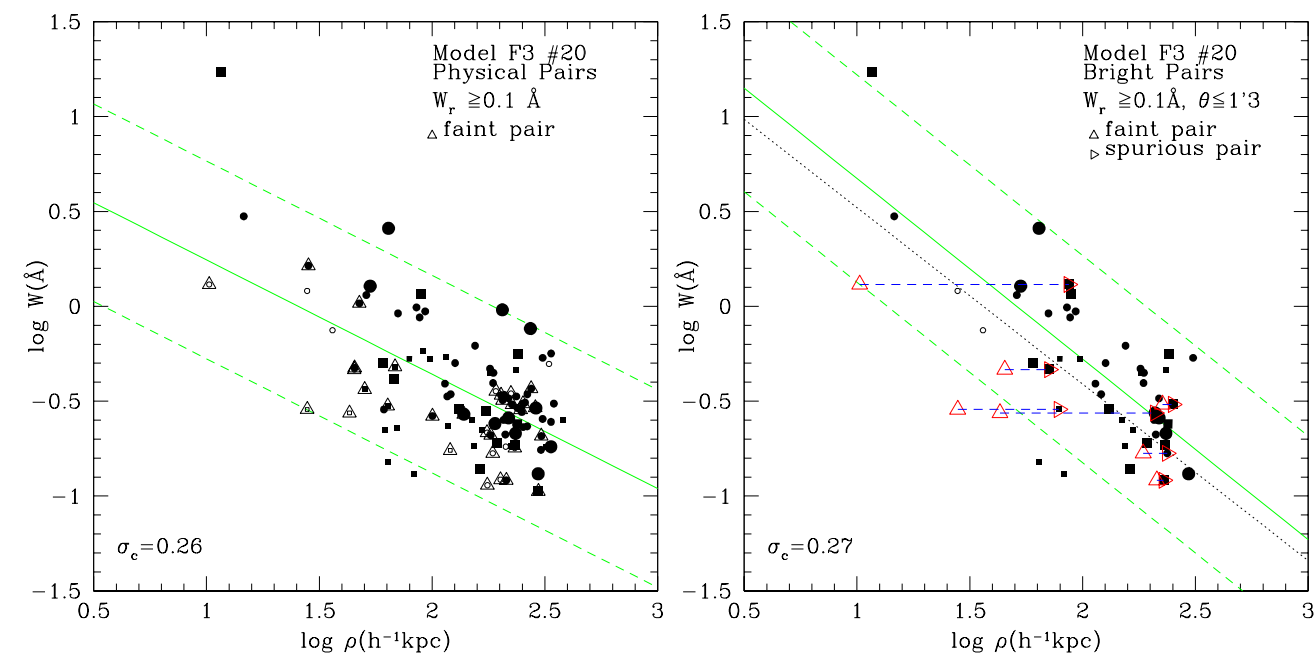

Figure 1. The anti-correlation of $W_{r}$ versus $\rho$ for run No. 20 of the mock data: the left and right panel are for 'physical pairs' and 'bright pairs' respectively. The horizontal long-dashed-lines represent the probability that a faint absorber (triangle at the left end of the line) may be mis-identified and paired with a nearby bright galaxy (triangle at the right end of the line). The dotted-line is the linear fit of Chen et al. (1998). The squares and circles are for spiral galaxies and elliptical/S0 galaxies respectively. Small open symbols represent galaxies of luminosity $L_{B} \leqslant 0.1 L_{B *}$, small filled symbols represent galaxies of luminosity $0.1 L_{B *}<L_{B} \leqslant L_{B *}$, large filled symbols represent galaxies of luminosity $L_{B}>L_{B *}$.

same redshift as the line. This absorber pair can only be classed as a candidate because a possible fainter galaxy at closer position could give rise to the absorption but escapes from the optical survey (Tripp et al. 1998; Impey et al. 1999). This can be simulated in so-called mock surveys. We made such mock surveys (100 runs) using the same set of QSOs (10 QSOs, with a total redshift interval of about 5) as in a real optical survey of absorbers (Chen et al. 1998) and applied similar criteria when selecting galaxy-absorber pairs. The mock observations predict about 26 bright absorber-galaxy pairs which is in good agreement with the results of real surveys (Chen et al. 1998). (See Lin et al. 2000 for more details on the comparisons of the statistical distribution of absorber properties such as REWs, luminosities, projected distances, apparent magnitudes, and redshifts with observational quantities.) As we have found, there indeed are some mis-identified pairs (We name these as "spurious pairs"). The mis-identification of absorber-galaxy pairs has significant impact on the analysis of relations between REW and projected distance, luminosity, and other results one can derive. On average, selection effects can strengthen the anti-correlation between REW and projected distance (together with the luminosity). Some specific runs of mock observations show agreement with observational results. Fig. 1 is an example of the relation between REW and $\rho$, and $L_{B} / L_{B *}$. This figure also shows how the mis-identification of absorber-galaxy pairs happens and affects the relation. There is a substantial number of missing pairs in the mock observations (see Fig. 8 of Lin et al. 2000).

Since there is large scatter in the results of each run of mock observations, we argue that the total redshift interval $(\sim 5)$ in contemporary optical surveys possibly is not large enough. In the mock surveys, an adequate redshift interval should be at least $\sim 10$ (and 
even $\sim 20$ ) for the above correlation analysis, as is shown by the statistical analysis of the results of mock observations.

\section{Strong absorption lines for MgII, SiII, and FeII}

The models can be applied to absorption line systems for photo-ionised ions such as Mg II , Si II , and Fe II. Here we only give results for Mg II absorption lines and present results for absorption lines arising from Si II and Fe II elsewhere (see Wang \& Lin 2005). Our model-predicted $d N / d z$ for $W_{\text {MgII }} \geqslant 0.3 \AA$ lines was about 0.51 (103 absorbers for $200 \mathrm{LsOS}$ ) averaged over the redshift span from 0 to 1 . The strong Mg II systems are generally thought to be the observational counterparts to the Lyman limit systems, for which $d N / d z=0.5 \pm 0.3$ at $z=0.5$ (Bahcall et al. 1996) and $d N / d z=0.7 \pm 0.2$ at $\langle z\rangle=0.7$ (Stengler-Larrea et al. 1995). Thus our predicted $d N / d z$ for strong $\mathrm{Mg}$ II systems is in good agreement with observational results. We also calculated the average $d N / d z$ for the redshift intervals from 0 to 0.5 and from 0.5 to 1.0. The numbers are 0.55 and 0.48 respectively. Therefore there is no strong redshift evolution of $d N / d z$ for strong Mg II systems in the simulation, in good agreement with observations.

There exists an anti-correlation between REW and projected distance, namely $\log W_{r}=$ $-\alpha \log \rho+$ constant. The analysis shows that $\alpha \simeq 0.38 \pm 0.03$ and the constant is $\sim$ $-0.05 \pm 0.05$. With all the simulated absorption-line galaxy pairs, the analysis of $\log W_{r}=$ $-\alpha \log \rho+\beta \log \left(L_{B} / L_{B *}\right)+$ constant, gives $\alpha=0.45 \pm 0.03, \beta=0.16 \pm 0.03$, and the constant is $0.14 \pm 0.06$. Thus the absorbing radius ( $\mathrm{REW} \geqslant 0.3 \AA$ ) of a galaxy with luminosity of $L_{B}$ will be $R_{a b s} \approx 28.7 h^{-1} \mathrm{kpc}\left(L_{B} / L_{B *}\right)^{0.35}$. The observational result is $R_{a b s} \approx 35 h^{-1} \operatorname{kpc}\left(L_{B} / L_{B *}\right)^{0.2}$ (Steidel 1995). If the selection criteria as in the last section are applied, the anti-correlation becomes stronger (Fig. 2) and the constant becomes $0.44 \pm 0.10$ for the same analysis. Furthermore the average absorbing radius of a galaxy with a luminosity of $L_{B}$ becomes $R_{a b s} \approx 37.9 h^{-1} \operatorname{kpc}\left(L_{B} / L_{B *}\right)^{0.18}$, which is consistent with observational results. This implies that the observational results so far could have been biased by selection effects.

Further improvements of the models are in progress. We are now considering spectrum resolution, signal-to-noise ratio, instrument profile, as well as collisional ionisation for highly-ionised species of ions. The simulated line profiles look like real ones (see Wang \& Lin 2005).

\section{Summary}

We present results of Monte-Carlo simulations of $\operatorname{Ly} \alpha$ and $\mathrm{Mg}$ II absorption line systems at redshift $z<1$. Full models with all three components (galaxy disk, cold clouds in a galactic halo, and satellites) can explain up to 60 percent of the observed number density of strong Ly $\alpha$ lines at low redshift. These models can also predict reasonable number densities of Lyman-limit systems, strong Mg II lines, and damped Ly $\alpha$ systems at low redshift. For correlation analysis, in general, if we assume $W_{r} \propto \rho^{-\alpha} L_{B}^{\beta}(1+z)^{-\gamma}$, the analysis gives $\alpha \sim 0.5, \beta \sim 0.15$, and $\gamma \sim 0.5$. The average covering factor within $250 h^{-1} \mathrm{kpc}$ is estimated as $\sim 0.36$ and the effective absorption radius is estimated to be $150 h^{-1} \mathrm{kpc}$, in good agreement with observational results (Chen et al. 1998). We constructed mock observations with the same known QSO LOS as Chen et al. (1998) and applied selection criteria which are similar. The predicted number of galaxy-absorber pairs with $W_{r} \geqslant 0.3 \AA$ is $\sim 26 \pm 5$, in good agreement with Chen et al. $(\sim 26)$. The analysis of the anti-correlation between $W_{r}$ and $\rho$ shows that selection effects can statistically strengthen the anti-correlation. We find the total redshift interval in present surveys is possibly not large enough to reveal the real anti-correlation. 

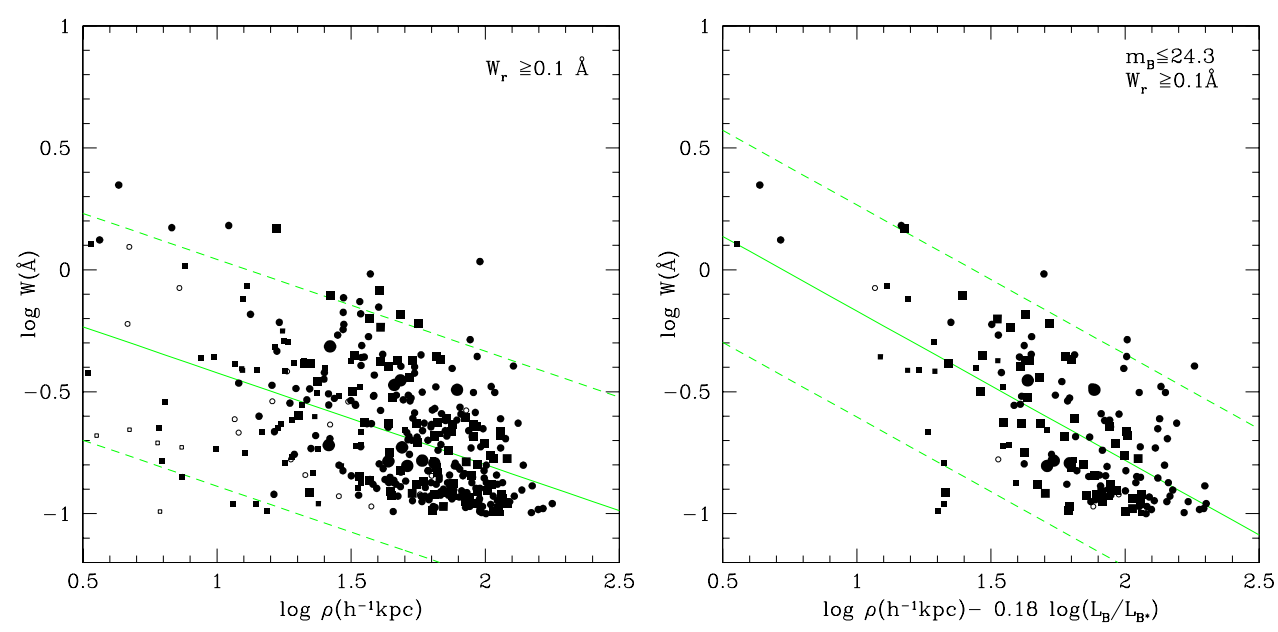

Figure 2. The analysis of $\log W_{r}=-\alpha \log \rho+\beta \log \left(L_{B} / L_{B}\right)+$ constant for galaxy-absorber pairs in the mock survey before (left panel) and after (right panel) taking into account selection effects. The lines and symbols are the same as in Fig. 1.

\section{Acknowledgements}

WPL was supported by NFSC No. 10203004, NKBRSF G1999075402, and Shanghai NFS No. 02ZA14093. WPL thanks the exchange program between CAS and MPG.

\section{References}

Bahcall, J. N., et al., 1996, ApJ, 457, 19

Chen, H.-W., Lanzetta, K. M., Webb, J. K., Barcons X., 1998, ApJ, 498, 77

Ellis, R. S., Colless, M., Broadhurst, T., Heyl, J., Glazebrook, K., 1996, MNRAS, 280, 235

Faber, S. M., Jackson, R. E., 1976, ApJ, 204, 668

Ferland, G. J., 1996, Hazy, a Brief Introduction to Cloudy, University of Kentucky, Department of Physics and Astronomy Internal Report.

Impey, C. D., Petry, C. E., Flint, K. P., 1999, ApJ, 524, 536

Klypin, A. A., Kravtsov, A. V., Valenzuela, O., Prada, F., 1999, ApJ, 522, 82

Lanzetta, K. M., Bowen, D. V., Tytler, D., Webb, J. K., 1995, ApJ, 442, 538

Lin, W. P., Börner, G., Mo, H. J., 2000, MNRAS, 319, 517.

Lin, W. P., Hong, B. H., 2002, Ch.Phys.Lett., 19, 10, 1565

Lin, W. P., Zou, Z. L., 2001, ChJA\&A, 1, 1, L21

Madau, P., 1992, ApJ, 389, L1

Miralda-Escudé, J., Ostriker, J. P., 1990, ApJ, 350, 1

Mo, H. J., 1994, MNRAS, 269, L49

Mo, H. J., Miralda-Escudé, J., 1996, MNRAS, 469, 589

Steidel, C. C., 1995, in QSO Absorption Lines, ed. G. Meylan (Berlin:Springer), p. 139

Stengler-Larrea, E. A., et al., 1995, ApJ, 444, 64

Tripp, T. M., Lu, L., Savage, B. D., 1998, ApJ, 508, 200

Tully, R. B., Fisher, J. R. 1977, A\&A, 54, 661

Wang, X. H., Lin, W. P., 2005, in preparation. 